\title{
GERENCIAMENTO DE ENFERMAGEM NO CONTROLE DE PERDAS EVITÁVEIS DE IMUNOBIOLÓGICOS
}

Vanessa Comassetto Oliveira', Cristiano Caveião², Fernanda Crosewski ${ }^{3}$

${ }^{1}$ Enfermeira. Doutoranda em Enfermagem. Professora das Faculdades Integradas do Brasil. Curitiba-PR-Brasil.

${ }^{2}$ Enfermeiro. Doutorando em Enfermagem. Professor das Faculdades Integradas do Brasil.. Curitiba-PR-Brasil.

${ }^{3}$ Enfermeira. Especialista em Enfermagem na Saúde da Família. Secretaria de Saúde do Estado do Paraná. Curitiba-PR-Brasil.

RESUMO: Os imunobiológicos são termolábeis, mantidos sob refrigeração desde o laboratório produtor até a sua administração. Este estudo teve como objetivos levantar o número e identificar as causas de perdas evitáveis de imunobiológicos no estado do Paraná, no período de 2009 a 2012. É um estudo exploratório retrospectivo quantitativo, cujos dados foram obtidos na base de dados do Sistema de Informação de Apuração de Imunobiológicos Utilizados do Paraná. O total das perdas de imunobiológicos por causas evitáveis foram de 3.437 .552 doses, sendo que $95 \%$ das perdas foram vacinas do calendário básico de vacinação. Pode-se identificar que ocorreram falhas na organização do serviço e/ou no processo de trabalho da enfermagem, por se tratar de perdas consideradas como evitáveis. O enfermeiro é o gerenciador das atividades de imunização, sendo imprescindível a atualização deste profissional, através de capacitações e educação continuada para um serviço eficaz no controle das doenças imunopreveníveis. DESCRITORES: Enfermagem; Vacinas; Refrigeração.

\section{NURSING MANAGEMENT IN THE CONTROL OF AVOIDABLE LOSSES OF IMMUNOBIOLOGICAL PREPARATIONS}

\begin{abstract}
Immunobiological preparations are thermolabile, requiring refrigeration from the production laboratory until their administration. This study aimed to quantify and identify the causes of avoidable losses of immunobiological preparations in Paraná state, for the period 2009 to 2012. This was a quantitative, retrospective, exploratory study, in which data were obtained using the database of the Information System for the Calculation of Immunobiological Preparations Used of Paraná. Total losses of immunobiological preparations due to avoidable causes were $3,437,552$ doses, with $95 \%$ of the losses being vaccines of the basic vaccination program. Failures that occurred in the organization of the service and/or the nursing work process can be identified, as the losses were considered preventable. The nurse is the manager of immunization activities, therefore, it is imperative to update this professional through training and continuing education for an effective service in the control of vaccinepreventable diseases.
\end{abstract}

KEYWORDS: Nursing; Vaccines; Refrigeration.

\section{ADMINISTRACIÓN DE ENFERMERÍA EN EL CONTROL DE PÉRDIDAS EVITABLES DE INMUNOBIOLÓGICOS}

RESUMEN: Los inmunobiológicos son termolables y se quedan bajo refrigeración desde el laboratorio productor hasta su administración. Este estudio tuvo como objetivos investigar el número e identificar las causas de pérdidas evitables de inmunobiológicos en el estado de Paraná, en el periodo de 2009 a 2012. Es un estudio exploratorio retrospectivo cuantitativo, cuyos datos fueron obtenidos en la base de datos del Sistema de Información de Apuración de Inmunobiológicos Utilizados de Paraná. El total de las pérdidas de inmunobiológicos por causas evitable fueron de 3.437.552 doses, siendo 95\% de las pérdidas de vacunas del calendario básico de vacunación. Se puede identificar que hubo fallas en la organización del servicio y/o en el proceso de trabajo de enfermería, ya que son pérdidas consideradas evitables. El enfermero es el administrador de las actividades de inmunización, siendo imprescindible la actualización de este profesional, por medio de capacitaciones y educación continuada para un servicio eficaz en el control de las enfermedades imunopreveibles. DESCRIPTORES: Enfermería; Vacunas; Refrigeración.
Autor Correspondente:

Vanessa Comassetto Oliveira

Faculdades Integradas do Brasil

Rua Major Vicente de Castro, 2138 - 81030-020 - Curitiba-PR-Brasil

E-mail: vancomassetto@hotmail.com
Recebido: $29 / 04 / 2014$

Finalizado: 20/09/2014 


\section{INTRODUÇÃO}

O Programa Nacional de Imunizações (PNI) foi instituído pelo Ministério da Saúde em 1973, com a finalidade de coordenar ações de imunização, utilizando estratégias básicas de vacinação, desenvolvidas de forma hierarquizada e descentralizada, contribuindo para o controle, eliminação e/ou erradicação das doenças imunopreveníveis ${ }^{(1)}$.

A Rede de frio é o processo de armazenamento, conservação, manipulação, distribuição e transporte dos imunobiológicos. O PNI garante as condições adequadas de refrigeração, desde o laboratório produtor até o momento em que o produto é administrado(1).

As vacinas representam a estratégia de intervenção com a melhor relação custo-benefício até hoje aplicada em saúde pública, seu impacto epidemiológico é fundamental na redução e controle das doenças imunopreveníveis ${ }^{(2)}$. Entretanto o uso crescente da utilização dos imunobiológicos traz consigo a necessidade de garantir a qualidade desses produtos.

Como produtos de origem biológica, os imunobiológicos são compostos por agentes imunizantes como as vacinas, os soros homólogos e os soros heterólogos. São produtos termolábeis, ou seja, quando expostos a temperaturas inadequadas sofrem inativação dos seus componentes imunogênicos, comprometendo sua potência e eficácia, uma vez que o calor acelera a inativação desses componentes. Assim sendo, os imunobiológicos devem ser conservados sob refrigeração, em todas as instâncias, conforme definido pelo laboratório produtor ${ }^{(3)}$.

O PNI conta com vários Sistemas de Informação, que permitem acompanhar, analisar e avaliar as Redes de Frio de todo o país, desde o estoque, distribuição, doses aplicadas, como também as perdas de imunobiológicos, sejam elas perdas técnicas ou perdas físicas, o que garante realizar um diagnóstico para organização e planejamentos de distribuições futuras ${ }^{(4)}$.

O controle das perdas é analisado por meio do Sistema de Informação de Apuração de Imunobiológicos Utilizados (SI-AIU), classificando os tipos de perdas. As perdas físicas, consideradas evitáveis, recebem a seguinte classificação: quebra de frasco - frascos ou ampolas de um imunobiológico que é quebrado; falta de energia elétrica - imunobiológico desprezado por exposição à temperatura inadequada, devido há interrupção no fornecimento de energia elétrica; falha no equipamento - ocorrência de falha mecânica no equipamento de refrigeração; validade vencida - expiração do prazo de validade informado no rótulo do produto; procedimento inadequado - perda de imunobiológico decorrente de erros de procedimentos técnicos padronizados; falha no transporte - falhas durante o transporte; outros motivos - imunobiológico perdido em decorrência de qualquer outro motivo ainda não relacionado anteriormente ${ }^{(4)}$.

Em 2011, o PNI publicou uma avaliação sistemática, realizada através do SI-AIU, com quatro estados do Brasil no ano de 2008, sobre o levantamento das principais causas de perdas de vacinas, e o custo financeiro associado a estas perdas. Os resultados mostraram que dois estados apresentaram uma média de $38 \%$ de perdas físicas classificadas por "falta de energia elétrica". Os outros dois estados apresentaram uma média de $39 \%$ de perdas físicas por "outros motivos", o que sugere um possível erro de registro no sistema uma vez que o mesmo não detalha este tipo de ocorrência. Quando avaliado o custo financeiro gerado pelas perdas dos quatro estados, este ficou próximo dos R\$ 8 milhões de reais, valor que poderia ser utilizado na aquisição de novos imunobiológicos ${ }^{(5)}$, e ações do programa.

Para que o PNI atinja seu principal objetivo, a redução de doenças imunopreveníveis, além da adesão da população, é fundamental garantir a qualidade dos imunobiológicos oferecidos à população, incluindo a conservação dos imunobiológicos, com o funcionamento adequado da Rede de Frio. Os profissionais de enfermagem são os mais envolvidos nesta área, uma vez que exercem atividades administrativas e assistenciais. São responsáveis pelas ações de imunização, cabendo-lhes garantir o processo de conservação dos imunobiológicos, sendo considerados aptos para tal atividade, através da legislação que regulamenta a prática de enfermagem $^{(6-7)}$.

No entanto, as equipes de enfermagem estão envolvidas com muitas ações administrativas, o que pode justificar as ocorrências de falhas no cumprimento das recomendações para conservação de imunobiológicos, podendo comprometer suas características imunogênicas, 
ocasionando a perda do produto ${ }^{(6)}$.

Estudos realizados no Brasil sobre a prática de enfermagem na conservação de vacinas mostram que muitos dos profissionais de enfermagem que atuam nesta área, desconhecem normas básicas, como a temperatura adequada para a conservação de vacinas nas unidades de saúde, ou ainda, sobre o tempo de validade dos imunobiológicos após abertura do frasco ${ }^{(6)}$. Reforçando a importância da educação permanente desses profissionais, uma vez que estas informações estão contidas nos manuais de rede de frio disponibilizados pelo PNI.

Diante do exposto, visto que o profissional de enfermagem é o responsável pelas ações de imunização, desde a conservação até a administração das vacinas, é relevante conhecer as causas das perdas evitáveis de imunobiológicos, tanto para a análise do processo de trabalho neste domínio, como para subsidiar o gerenciamento da rede de frio, buscando minimizar as perdas vacinais.

Nesse sentido este trabalho tem como objetivos, levantar o número e identificar as causas de perdas evitáveis de imunobiológicos no estado do Paraná no período de 2009 a 2012.

\section{MÉTODO}

Trata-se de uma pesquisa exploratória, retrospectiva, de caráter quantitativo. Este tipo de pesquisa tem como objetivo proporcionar maior familiaridade com o problema, com vistas a tornálo mais explícito e aprimorar ideias ainda pouco exploradas. Seu planejamento é bastante flexível, de modo que possibilite a consideração dos mais variados aspectos relativos ao fato estudado ${ }^{(8)}$.

O estado do Paraná está dividido administrativamente em 22 Regionais de Saúde (RS) e 399 municípios, sendo que todas as
RS possuem uma rede de frio, a qual fornece imunobiológicos para os municípios de sua abrangência. Da mesma forma, cada município possui uma rede de frio, a qual distribui imunobiológicos para suas Unidades de Saúde de abrangência ${ }^{(9-10)}$.

Os dados para esta pesquisa foram obtidos no SI-AIU do estado do Paraná. Foram selecionados os relatórios de Movimentação de Imunobiológicos na instância das 22 redes de frio Regionais, 399 redes de frio Municipal e também as salas de vacinas, dentre os anos de 2009 a 2012.

Foram incluídos todos os imunobiológicos que constam no SI-IAU do estado do Paraná, ou seja, vacinas e diluentes soros homólogos e heterólogos, distribuídos pelo PNI.

A coleta de dados foi realizada entre os meses de janeiro e fevereiro de 2013, e para tal, foi construído um instrumento em planilha Excel que possibilitou a organização e análise dos dados. A solicitação para acesso ao banco de dados foi autorizada pela Coordenação Estadual de Imunização da Secretaria do Estado da Saúde do Paraná.

\section{RESULTADOS}

Os dados obtidos mostram que no Paraná as perdas de imunobiológicos por causas evitáveis foram de 3.437.552 doses no período de 2009 a 2012. O ano de 2011 foi o que mais se destacou, com $38 \%$ do total de doses perdidas neste período.

Do total de perdas, $1,5 \%$ das doses foram por Quebra de Frasco (QF); 7\% por Falta de Energia (FE); 9\% por Falha no Equipamento (FEq); 76,5\% por Validade Vencida (VV); $2 \%$ por Procedimento Inadequado (PI); $0,4 \%$ por Falha no Transporte (FT); e 4\% doses por Outros motivos, conforme Tabela 1.

Tabela 1 - Discriminação do total de perdas de imunobiológico, no período de 2009 a 2012. Paraná-Brasil, 2013

\begin{tabular}{lcccccccc}
\hline Ano & QF & FE & FEq & VV & PI & FT & Outras & Total \\
\hline 2009 & 4.508 & 48.170 & 31.525 & 410.842 & 9.294 & 4.217 & 33.683 & 542.239 \\
\hline 2010 & 6.033 & 64.660 & 62.363 & 520.226 & 8.575 & 1.783 & 24.659 & 688.299 \\
\hline 2011 & 35.342 & 76.705 & 131.786 & 972.584 & 27.830 & 7.419 & 60.093 & 1.311 .759 \\
\hline 2012 & 6.899 & 41.506 & 73.247 & 727.791 & 12.001 & 1.079 & 32.732 & 895.255 \\
\hline Total & 52.782 & 231.041 & 298.921 & 2.631 .443 & 57.700 & 14.498 & 151.167 & 3.437 .552 \\
\hline
\end{tabular}

Legenda: QF: Quebra de Frasco; FE: Falta de Energia; FEq: Falha no Equipamento; VV: Validade Vencida; PI: Procedimento Inadequado; FT: Falha no Transporte.

Fonte: SI-AIU/PNI/MS - PR. 
A discriminação das perdas evitáveis das vacinas do calendário básico de vacinação (Tabela 2) foram analisadas separadamente dos imunobiológicos especiais (Tabela 3).

Os imunobiológicos que mais apresentaram ocorrências foram as vacinas do calendário básico de vacinação, administradas na rotina dos serviços de saúde, representando 3.273.861 (95,2\%) doses.

Destaca-se a perda por Validade Vencida, foi responsável pela perda de 2.489.337 (76\%) doses destas vacinas. Na sequência, a perda por Falha no Equipamento de refrigeração com 292.472 (8,9\%) doses. A Falta de Energia elétrica representa 227.048 (6,9\%) destas doses. A perda por Outros motivos foi significativa, com 144.002 $(4,4 \%)$ doses. O Procedimento Inadequado apresentou a perda de $56.273(1,7 \%)$ doses. A
Quebra de Frasco também aparece como perda evitável com 51.087 (1,6\%) doses. E a Falha no Transporte com 13.642 (0,4\%).

De acordo com Tabela 3, os imunobiológicos especiais apresentaram 163.691 (4,8\%) do total de doses perdidas, e assim como as vacinas de rotina, a principal causa foi a Validade Vencida, representando 142.106 (86,8\%) doses. A segunda maior causa de perda foi por Outros motivos com 7.165 (4,4\%) doses. A Falha no Equipamento aparece com perda de 6.449 (3,9\%) doses. Estes também apresentaram perda por Falta de Energia elétrica com 3.993 (2,4\%) doses. A perda dos imunobiológicos especiais por Quebra de Frasco representa $1.695(1,0 \%)$ doses. O Procedimento Inadequado com 1.427 (0,9\%) doses perdidas e a Falha no Transporte com 856 (0,5\%) doses.

Tabela 2 - Discriminação das perdas de vacinas do esquema básico de vacinação, no período de 2009 a 2012. Paraná-Brasil, 2013

\begin{tabular}{|c|c|c|c|c|c|c|c|c|}
\hline Vacina & QF & FE & FEq & VV & PI & FT & Outras & Total \\
\hline $\begin{array}{l}\text { Tríplice Bact. } \\
\text { (DTP) }\end{array}$ & 1.911 & 12.160 & 19.590 & 308.320 & 2.690 & 540 & 10.680 & 355.891 \\
\hline $\begin{array}{l}\text { Hepatite B } \\
\text { (HB) }\end{array}$ & 3.570 & 18.634 & 29.075 & 232.676 & 4.875 & 2360 & 11.975 & 303.165 \\
\hline $\begin{array}{l}\text { Febre amarela } \\
\text { (FA) }\end{array}$ & 1.345 & 41.510 & 39.075 & 452.690 & 7.045 & 1535 & 15.545 & 558.745 \\
\hline BCG & 4.680 & 14.800 & 28.980 & 208.080 & 4.730 & 450 & 10.520 & 272.240 \\
\hline $\begin{array}{l}\text { Polio inativada } \\
\text { (Salk) }\end{array}$ & 210 & 781 & 415 & 3.667 & 121 & 30 & 608 & 5.832 \\
\hline $\begin{array}{l}\text { Tríplice viral } \\
\text { (SCR) }\end{array}$ & 3.424 & 27.663 & 23.549 & 101.400 & 4.039 & 1461 & 17.061 & 178.597 \\
\hline $\begin{array}{l}\text { Dupla adulto } \\
\text { (dT) }\end{array}$ & 3.030 & 19.968 & 41.655 & 375.901 & 4.630 & 580 & 16.555 & 462.319 \\
\hline $\begin{array}{l}\text { Polio atenuada } \\
\text { (Sabin) }\end{array}$ & 28.895 & 51.985 & 59.020 & 378.200 & 17.275 & 5280 & 45.230 & 585.885 \\
\hline $\begin{array}{l}\text { Dupla viral } \\
\text { (SR) }\end{array}$ & 430 & 10.180 & 3.890 & 193.530 & 370 & 90 & 1.341 & 209.831 \\
\hline $\begin{array}{l}\text { Raiva - cel. } \\
\text { Vero }\end{array}$ & 674 & 1.791 & 1.867 & 8.120 & 580 & 150 & 1.060 & 14.242 \\
\hline $\begin{array}{l}\text { Tetra valente } \\
(\mathrm{DTP}+\mathrm{Hib})\end{array}$ & 1.455 & 12.886 & 24.393 & 41.784 & 4.124 & 593 & 7.160 & 92.385 \\
\hline Meningo. C & 317 & 6.296 & 4.166 & 1.272 & 823 & 92 & 1.264 & 14.230 \\
\hline $\begin{array}{l}\text { Penta valente } \\
\mathrm{DTP}+\mathrm{Hib}+\mathrm{HB}\end{array}$ & 38 & 286 & 207 & 12.393 & 85 & 06 & 33 & 13.048 \\
\hline $\begin{array}{l}\text { Rotavírus } \\
\text { hum. (VORH) }\end{array}$ & 641 & 3.939 & 8.717 & 170.876 & 3.748 & 333 & 3.291 & 191.545 \\
\hline $\begin{array}{l}\text { Pneumo. } 10 \\
\text { val. (Pnm 10) }\end{array}$ & 467 & 4.169 & 7.873 & 428 & 1.138 & 152 & 1.679 & 15.906 \\
\hline Total & 51.087 & 227.048 & 292.472 & 2.489 .337 & 56.273 & 13.642 & 144.002 & 3.273 .861 \\
\hline
\end{tabular}

Fonte: SI-AIU/PNI/MS - PR. 
Tabela 3 - Discriminação das perdas de imunobiológicos especiais, 2009 a 2012. Paraná-Brasil, 2013

\begin{tabular}{|c|c|c|c|c|c|c|c|c|}
\hline Imunobiológico & QF & $\mathrm{FE}$ & FEq & VV & PI & FT & Outras & Total \\
\hline $\begin{array}{l}\text { Imunog. } \\
\text { antitetânica }\end{array}$ & 3 & 14 & 15 & 1.573 & 7 & 0 & 2.401 & 4.013 \\
\hline $\begin{array}{l}\text { Soro an- } \\
\text { titetânico }\end{array}$ & 91 & 119 & 31 & 1.406 & 21 & 0 & 45 & 1.713 \\
\hline $\begin{array}{l}\text { Soro anti- } \\
\text { aracnídeo }\end{array}$ & 44 & 29 & 61 & 734 & 12 & 0 & 15 & 895 \\
\hline $\begin{array}{l}\text { Soro anti- } \\
\text { escorpiônico }\end{array}$ & 19 & 57 & 21 & 696 & 9 & 0 & 10 & 812 \\
\hline $\begin{array}{l}\text { Soro anti- } \\
\text { elapídico }\end{array}$ & 8 & 5 & 0 & 429 & 5 & 0 & 10 & 457 \\
\hline $\begin{array}{l}\text { Soro anti } \\
\text { rábico }\end{array}$ & 118 & 69 & 87 & 1.080 & 42 & 1 & 129 & 1.526 \\
\hline $\begin{array}{l}\text { Soro anti- } \\
\text { botrópico }\end{array}$ & 104 & 108 & 119 & 6.293 & 25 & 6 & 148 & 6.803 \\
\hline $\begin{array}{l}\text { Soro an- } \\
\text { tidiftérico }\end{array}$ & 3 & 0 & 0 & 187 & 0 & 0 & 0 & 190 \\
\hline $\begin{array}{l}\text { Soro botróp/ } \\
\text { crotál. }\end{array}$ & 4 & 10 & 2 & 94 & 0 & 0 & 7 & 117 \\
\hline $\begin{array}{l}\text { Soro anti- } \\
\text { crotálico }\end{array}$ & 51 & 84 & 101 & 1.580 & 14 & 0 & 37 & 1.867 \\
\hline $\begin{array}{l}\text { Haemophilus } \\
\text { inf. B }\end{array}$ & 5 & 1 & 15 & 1.498 & 0 & 5 & 17 & 1.541 \\
\hline $\begin{array}{l}\text { Imunog. anti } \\
\text { varicela }\end{array}$ & 0 & 0 & 0 & 159 & 0 & 0 & 5 & 164 \\
\hline $\begin{array}{l}\text { Imunog. anti } \\
\text { hepat. B }\end{array}$ & 5 & 14 & 7 & 1.282 & 2 & 5 & 21 & 1.336 \\
\hline $\begin{array}{l}\text { Pneumocócica } \\
23 \mathrm{val}\end{array}$ & 6 & 136 & 168 & 2.905 & 27 & 11 & 85 & 3.338 \\
\hline $\begin{array}{l}\text { Imunog. anti } \\
\text { rábica }\end{array}$ & 0 & 0 & 8 & 377 & 0 & 10 & 17 & 412 \\
\hline Febre tifoide & 10 & 0 & 0 & 121 & 11 & 0 & 6 & 148 \\
\hline $\begin{array}{l}\text { Soro anti- } \\
\text { loxoscélico }\end{array}$ & 1 & 5 & 18 & 642 & 14 & 0 & 12 & 692 \\
\hline $\begin{array}{l}\text { Soro anti- } \\
\text { lonômia }\end{array}$ & 0 & 5 & 10 & 682 & 0 & 0 & 0 & 697 \\
\hline Influenza & 709 & 2.824 & 2.023 & 60.104 & 645 & 610 & 3.405 & 70.320 \\
\hline DTP acelular & 2 & 3 & 6 & 272 & 0 & 1 & 5 & 289 \\
\hline Varicela & 25 & 47 & 126 & 538 & 19 & 7 & 4 & 766 \\
\hline Hepatite A & 3 & 6 & 14 & 109 & 0 & 0 & 7 & 139 \\
\hline $\begin{array}{l}\text { Soro antibu- } \\
\text { tolínico }\end{array}$ & 15 & 10 & 40 & 305 & 145 & 0 & 230 & 745 \\
\hline $\begin{array}{l}\text { Pneumocócica } \\
7 \mathrm{val}\end{array}$ & 9 & 170 & 61 & 91 & 15 & 0 & 31 & 377 \\
\hline $\begin{array}{l}\text { Influenza } \\
\text { H1N1 }\end{array}$ & 460 & 277 & 3.516 & 58.949 & 414 & 200 & 518 & 64.334 \\
\hline Total & 1.695 & 3.993 & 6.449 & 142.106 & 1.427 & 856 & 7.165 & 163.691 \\
\hline
\end{tabular}

Fonte: SI-AIU/PNI/MS - PR. 


\section{DISCUSSÃO}

A fim de facilitar a compreensão dos achados serão analisados primeiramente os motivos das perdas de vacinas do calendário básico, conforme demonstrado na Tabela 2, na sequência serão abordados os motivos das perdas das vacinas especiais, de acordo com a Tabela 3.

Destaca-se a perda por Validade Vencida, a qual corresponde a $76 \%$ de todas as perdas de vacinas de rotina, sendo que o imunobiológico que mais apresentou essa ocorrência foi a vacina febre amarela com 452.690 doses, e a vacina oral poliomielite (VOP) com 378.200 doses.

Evidenciou-se que em todos os outros motivos a vacina oral da poliomielite, comparada com os outros imunobiológicos da rotina, apresentou o maior número de doses perdidas, inclusive por Quebra de Frasco. No ano de 2011 o Paraná recebeu a vacina do laboratório Glaxo Smith Kline (GSK) com enfrascagem de vidro ${ }^{(11)}$, o que acarretou em um grande número de quebra de frasco operacional.

A otimização e a oferta das vacinas devem ser reforçadas constantemente, principalmente quando há um lote próximo do vencimento, que pode ser verificado no momento da organização do refrigerador, após a sua limpeza, preconizada a cada quinze dias e na elaboração do relatório mensal de estoque de imunobiológicos ${ }^{(1)}$. Esta atividade é de competência da equipe de enfermagem, devendo-se, portanto, questionar a rotina de serviços das Unidades de Saúde a fim de reduzir perdas evitáveis, o que vai ao encontro de resultados de estudos que apontam as dificuldades para manter a organização do refrigerador, tais como: estrutura inadequada, capacitação profissional insuficiente ou inexistente e alta rotatividade de profissionais atuantes na sala de vacinas ${ }^{(12)}$.

A Falha no Equipamento de refrigeração representa o segundo maior motivo de perdas das vacinais do calendário básico de vacinação. Este tipo de perda pode ser reduzida com a realização da manutenção preventiva e corretiva do refrigerador, substituição dos equipamentos antigos do tipo doméstico, por refrigeradores específicos para armazenamento de imunobiológicos ${ }^{(13)}$. O monitoramento diário da temperatura do refrigerador também é uma forma de avaliar se o equipamento está apresentando problemas $^{(1)}$. Este tipo de ocorrência pode acarretar em grandes oscilações de temperaturas, podendo comprometer a capacidade imunogênica das vacinas, pois vacinas de vírus vivo atenuado são sensíveis a temperaturas altas $\left(>8^{\circ} \mathrm{C}\right)$, já as de vírus inativados, e as vacinas bacterianas, são sensíveis a baixas temperaturas $\left(<2^{\circ} \mathrm{C}\right)^{(10)}$.

A Falta de Energia também foi evidenciada. Quando ocorrer a falta de energia elétrica o recomendado pelo $\mathrm{PNI}$ é que se mantenha o refrigerador fechado e que seja monitorada rigorosamente a temperatura interna, através de termômetro de máxima e mínima, até o prazo de duas horas. Caso a energia não volte, a equipe deve preparar caixas térmicas ambientadas com bobinas de gelo reciclável para uma possível transferência dos imunobiológicos do refrigerador para garantir que estes não sofram alteração de temperatura. Em alguns casos, o imunobiológico deverá ser remanejado para outra Rede de Frio (outra unidade ou secretaria) (1), até o reestabelecimento do problema.

Como o refrigerador do tipo doméstico não atende os critérios de segurança e qualidade na estabilização da temperatura, estes devem ser substituídos por câmaras refrigeradas específicas para esta finalidade. Estas câmaras quando comparadas ao refrigeradores domésticos, mantém a temperatura preconizada por um maior período, quando ocorre a falta de energia elétrica, possibilitando ao serviço, maior tempo para uma ação adequada ${ }^{(1-10)}$.

Quando analisadas as perdas dos imunobiológicos especiais observa-se que apresentaram perda significativa por Validade Vencida, correspondendo a $87 \%$, sendo a vacina influenza a que apresentou maior ocorrência por este motivo com 60.104 (36,7\%) doses, em relação aos outros imunobiológicos especiais.

As perdas relacionadas as vacinas oral poliomielite e a influenza, provavelmente são decorrentes das campanhas anuais de vacinação, onde existe uma maior quantidade de imunobiológico disponível no serviço de saúde para atender o publico alvo, e que as ocorrências podem ter coincidido com este período. Outra possível causa de perda pode estar associada ao não alcance das coberturas vacinais, ou ainda, há solicitação de doses a mais que o necessário, acarretando em estoque de vacinas que pode propiciar o vencimento antes da utilização. Por 
se tratar de uma demanda sazonal (período de campanha), este produto requer um controle diferenciado dos produtos permanentes ${ }^{(13)}$, ou seja, manter quantidades suficientes somente para atender a população estimada a ser vacinada, dentro daquele período.

Destaca-se também a perda por Outros motivos que representam 7165 (4,4\%) doses. As perdas por outros motivos não são detalhados nos sistemas de informação por se tratarem de frascos sem rótulo, impossibilitando a identificação do produto, liófilos sem diluentes (sem condição de reposição), com alterações de cor, presença de corpos estranhos, furtos e roubos. Entretanto observa-se um número elevado por estas perdas que deveriam ser "insignificantes", principalmente devido ao controle rigoroso de qualidade. Tal número vai de encontro com este fator, sugerindo falhas nos registros no SI-AIU.

A ocorrência por Falha no Equipamento também resultou em perda dos imunobiológicos especiais, com 6.449 (3,9\%) doses. Esta evidência reforça a necessidade de manter um contrato de manutenção preventiva e corretiva atualizado e renovado com antecedência, evitando períodos sem cobertura ${ }^{(1)}$.

Entende-se que a Quebra de Frasco pode ser evitada com a organização do serviço, pois o acondicionamento de forma adequada pode facilitar o manuseio dos frascos sob refrigeração, sem que ocorram acidentes ${ }^{(1)}$. Estudos com métodos avaliativos aplicados na sala de vacinação, observam que a falta da organização interna do refrigerador é visto com freqüência, o que pode contribuir para a quebra dos frascos dessas vacinas ${ }^{(14)}$.

Outro motivo de perda refere-se ao Procedimento Inadequado, ou seja, erros de procedimentos técnicos padronizados. Esta padronização é encontrada nos manuais de rede de frio ${ }^{(1)}$ e manual de normas de vacinação ${ }^{(3)}$, os quais são disponibilizados pelo $\mathrm{PNI}$ a todas as unidades de saúde. Estudos realizados no Brasil identificam que a conduta dos funcionários da sala de vacinação ainda deixam muito a desejar ${ }^{(10)}$, com isso, percebe-se a relevância dos treinamentos contidos nos manuais, além das supervisões e monitoramento do processo, pois há necessidade de reduzir ainda mais tais perdas, observadas nos resultados como terceira menor causa de perda dos imunobiologicos de rotina, e a segunda menor causa quanto aos especiais.

Em relação a todas as perdas identificadas, a menor equivale a Falha no Transporte. Essas falhas equivalem a motivos como esquecimento de caixas de imubiológicos no veículo, acidentes de trânsito, desvios de rota causando maior tempo de trajeto, entre outros. Os responsáveis por essa atividade são motoristas das secretarias municipais de saúde, os quais devido a sua função, provavelmente não recebem treinamento específico para transporte e conservação dos imunobiológicos.

Vale ressaltar que as vacinas do calendário básico de vacinação apresentaram maior número de doses perdidas quando comparadas aos imunobiológicos especiais. Entende-se que se deve ao fato de que no serviço de saúde a quantidade disponível das vacinas de rotina para atender toda a população é muito maior do que as vacinas especiais, que são disponibilizadas para administração nos serviços de saúde quando há uma solicitação médica, ou seja, quando há uma indicação especial para sua utilização, ou ainda em situações de emergência, como a utilização das imunoglobulinas e dos soros heterólogos.

Os resultados obtidos com esta pesquisa são preocupantes. A perda por causas evitáveis de imunobiológicos reflete a organização do serviço de saúde, no qual o enfermeiro é o responsável pelo gerenciamento.

Os imunobiológicos são produtos de alto custo para saúde pública e a sua perda significa não somente a perda financeira, mas pode representar o desabastecimento para atender a população e manter controladas as doenças imunopreveníveis.

\section{CONSIDERAÇÕES FINAIS}

A amplitude e o dinamismo do Programa de Imunização requerem atualizações constantes dos profissionais responsáveis pela rede de frio dos imunobiológicos. A cada ano novas vacinas são introduzidas no calendário de vacinação o que requer total atenção no gerenciamento destes imunobiológicos.

A pesquisa mostra que no Paraná e na maioria dos outros estados, o enfermeiro é o principal profissional atuante na rede de frio dos imunobiológicos, e que a organização do serviço 
e o processo de trabalho da enfermagem estão diretamente relacionados aos fatores responsáveis pelas perdas de imunobiológicos por causas evitáveis.

Dessa forma, acredita-se que a educação permanente dos profissionais envolvidos tem um grande potencial de mudanças necessárias, tanto para a redução das perdas evitáveis como principalmente para assegurar a qualidade dos imunobiológicos disponibilizados.

Esta pesquisa mostra o motivo pelo qual se perdem imunobiológicos. Os resultados poderão subsidiar os enfermeiros no gerenciamento adequado da rede de frio dos imunobiológicos, buscando minimizar as perdas evitáveis de vacinas.

\section{REFERÊNCIAS}

1. Ministério da Saúde (BR). Fundação Nacional de Saúde. Manual de rede de frio. $4^{a}$ ed. FUNASA. Brasília; 2013.

2. Ponte CF. Vacinação, controle de qualidade e produção de vacinas no Brasil a partir de 1960. Hist. cienc.saude. [Internet] 2003;10 Suppl 2 [acesso em 9 set 2012]. Disponível: http://dx.doi.org/10.1590/S010459702003000500009

3. Ministério da Saúde (BR). Fundação Nacional de Saúde. Manual de Normas de Vacinação. Brasília; 2001.

4. Ministério da Saúde (BR) [Internet]. Sistema de Informação do Programa Nacional de Imunizações SI-PNI. [acesso em 09 setembro 2012]. Disponível: http://pni.datasus.gov.br/apresentacao.asp

5. Samad S. La experencia de Brasil com el desarrollo de um sistema para evaluar la perdida de vacunas. Boletim de Imunização. Organización Panamericana de la Salud. Fevereiro; 2012

6. Oliveira VC, Guimarães EAA, Guimarães IA, Januário LH, Ponto IV. Prática de enfermagem na conservação de vacinas. Acta Paul. Enferm. [Internet] 2009;22(6) [acesso em 10 set 2012]. Disponível: http://dx.doi. org/10.1590/S0103-21002009000600014

7. Aranda CMSS, Moraes JC. Rede de frio para a conservação de vacinas em unidades públicas do município de São Paulo: conhecimento e prática. Rev. bras. Epidemiol.. [Internet] 2006;9(2) [acesso em 10 set 2012]. Disponível: http://dx.doi.org/10.1590/S1415790X2006000200004

8. Gil AC. Como elaborar projeto de pesquisa. $4^{\mathrm{a}}$ ed. São Paulo: Atlas, 2007. p. 41.
9. Secretaria de Estado da Saúde (PR)/Regionais de Saúde [internet]. Curitiba: Secretaria de Saúde do Estado do Paraná; [acesso em 10 set 2012]. Disponível em: www. sesa.pr.gov.br

10. Luna GLM, Vieira LJS, Souza PF, Lira SVG, Moreira DP, Pereira AS. Aspectos relacionados à administração e conservação de vacinas em centros de saúde no Nordeste do Brasil. Cienc. saude colet. [Internet] 2011; 16(2) [acesso em 10 fev 2012]. Disponível: http://dx.doi. org/10.1590/S1413-81232011000200014

11. Ministério da Saúde (BR). Manual do Sistema de Informação do Programa Nacional de Imunização. 2012.

12. Oliveira VC, Gallardo MDPS, Arcêncio RA, Gontijo TL, Pinto IC. Avaliação da qualidade de conservação das vacinas na atenção primária à saúde. Cienc. saude colet.. [Internet]. 2014; 19(9) [acesso em 18 set 2014]. Disponível em: http://dx.doi.org/10.1590/141381232014199.12252013

13. Carr C, Byles J, Durrheim D. Practice nurses best protect the vaccine cold chain in general practice. Aust. j. adv. nurs. [Internet]. 2010 27(2) [acesso em 18 set 2014]. Disponível em: http://www.ajan.com.au/ Vol27/Carr.pdf

14. Araújo ACM, Silva MRF, Frias PG. Avaliação da Rede de Frio do Programa Municipal de Imunização do Distrito Sanitário IV do Município do Recife. Rev. APS [Internet] 2009;12(3) [acesso em 18 set 2014]. Disponível em: http://aps.ufjf.emnuvens.com.br/aps/ article/view/438 